\title{
Which Schubert varieties are local complete intersections?
}

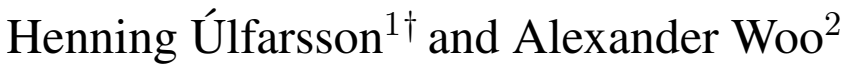 \\ ${ }^{1}$ School of Computer Science, Reykjavík University, Iceland \\ ${ }^{2}$ Department of Mathematics, University of Idaho, Moscow, ID, USA
}

\begin{abstract}
We characterize by pattern avoidance the Schubert varieties for $\mathrm{GL}_{n}$ which are local complete intersections (lci). For those Schubert varieties which are local complete intersections, we give an explicit minimal set of equations cutting out their neighborhoods at the identity. Although the statement of our characterization only requires ordinary pattern avoidance, showing that the Schubert varieties not satisfying our conditions are not lci appears to require working with more general notions of pattern avoidance. The Schubert varieties defined by inclusions, originally introduced by Gasharov and Reiner, turn out to be an important subclass, and we further develop some of their combinatorics. One application is a new formula for certain specializations of Schubert polynomials.

Résumé. Nous caractérisons par l'évitement des motifs les variétés de Schubert qui sont localement des intersections complètes. Pour les variétés de Schubert qui sont localement des intersections completes, nous donnons des ensembles explicites des polynômes qui définissent leurs voisinages à l'identité. Bien que notre caractérisation n'utilise que les motifs ordinare, nous avons besoin des notions plus générales des motifs dans notre démonstration. Les variétés de Schubert définies par des inclusions, introduites par Gasharov et Reiner, sont une sous-classe importante, et nous développons davantage leurs combinatoire. Une application est une nouvelle formule pour une specialisation des polynômes de Schubert.
\end{abstract}

Keywords: Schubert Varieties, Permutation Patterns

\section{Introduction}

This is an shortened version with details omitted of the paper [UW11], which has been submitted for publication elsewhere. The main goal is to classify by pattern avoidance the permutations $w$ for which the Schubert variety $X_{w}$ is a local complete intersection.

Let $G=\mathrm{GL}_{n}(\mathbb{C})$ and $B$ a Borel subgroup, which we take to be the upper triangular matrices. The quotient $G / B$ is a projective variety known as the flag variety; its points correspond to complete flags, which are chains of subspaces $F_{\bullet}=\langle 0\rangle \subsetneq F_{1} \subsetneq \cdots \subsetneq F_{n-1} \subsetneq \mathbb{C}^{n}$ with $\operatorname{dim} F_{i}=i$ for all $i$. The group $G$, and hence its subgroup $B$, acts on $G / B$ by left multiplication. Given a permutation $w$, the Schubert variety $X_{w}$ is the closure of the orbit $B w B / B$ of the permutation matrix for $w$ under the action of $B$.

†Úlfarsson was supported by grant no. 090038013 from the Icelandic Research Fund.

1365-8050 (c) 2012 Discrete Mathematics and Theoretical Computer Science (DMTCS), Nancy, France 
A local ring $R$ is a local complete intersection (lci) if it is the quotient of some regular local ring by an ideal generated by a regular sequence. A variety (or, more generally, a scheme) is lci if every local ring is lci. Smooth varieties are automatically lci, and lci varieties are automatically Gorenstein and hence Cohen-Macaulay. Thus, being lci can be viewed as saying that the singularities are in some sense mild.

Lakshmibai and Sandhya [LS90] found to some amazement at the time that smoothness of the Schubert variety $X_{w}$ can be characterized by the combinatorial notion of pattern avoidance. A permutation $v \in$ $S_{m}$ embeds in $w \in S_{n}$ if there are some $m$ entries of $w$, say at indices $i_{1}<\cdots<i_{m}$, in the relative order given by $v$, meaning that $w\left(i_{j}\right)<w\left(i_{k}\right)$ if and only if $v(j)<v(k)$. If $v$ does not embed in $w$, then $w$ is said to avoid $v$. Lakshmibai and Sandhya showed that $X_{w}$ is smooth if and only if $w$ avoids both of the permutations 3412 and 4231 (written in 1-line notation).

More recently, Yong and the second author characterized the permutations $w$ for which $X_{w}$ is Gorenstein [WY06]. This characterization cannot be given purely in terms of pattern avoidance but requires a more complicated generalization, either interval pattern avoidance (called Bruhat-restricted pattern avoidance in the original) or alternatively bivincular patterns as explained in [Ú11]. However, the lci Schubert varieties can be characterized by ordinary pattern avoidance. More precisely, we prove the following theorem.

Theorem 1.1 The Schubert variety $X_{w}$ is lci if and only if $w$ avoids the six patterns 53241, 52341, 52431, 35142,42513 , and 351624 .

For convenience we work over $\mathbb{C}$ in this paper, but our results and proofs hold over $\mathbb{Z}$ and hence over any field.

A further related result is the characterization of Schubert varieties which are defined by inclusions, due to Gasharov and Reiner [GR02]. They show that $X_{w}$ is defined by inclusions if $w$ avoids 4231, 35142, 42513 , and 351624. As one can tell from the patterns involved, our theorem implies that Schubert varieties defined by inclusions are lci, which was previously unknown. Indeed, the Schubert varieties defined by inclusions turn out to be an important special case in proving the sufficiency of our pattern avoidance conditions. In particular, we use Fulton's essential set [Ful92] to canonically associate a permutation defined by inclusions to any permutation indexing an lci Schubert variety.

More recently, Hultman, Linusson, Shareshian, and Sjöstrand [HLSS09] showed that, given a permutation $w$, the number of chambers in the inversion arrangement for $w$ is equal to the number of permutations less than or equal to $w$ in Bruhat order if and only if $w$ avoids the same patterns 4231, 35142, 42513, and 351624. The connection between this result and that of Gasharov and Reiner is at present a complete mystery. We hope our work may help in finding a connection.

\section{Schubert and Kazhdan-Lusztig varieties}

We briefly define Schubert varieties. A (complete) flag $F_{\bullet}$ in $\mathbb{C}^{n}$ is a sequence of subspaces $\langle 0\rangle \subsetneq F_{1} \subsetneq$ $F_{2} \subsetneq \cdots \subsetneq F_{n-1} \subsetneq F_{n}=\mathbb{C}^{n}$, with $\operatorname{dim} F_{i}=i$. As a set, the flag variety $\mathcal{F}_{n}$ has one point for every flag in $\mathbb{C}^{n}$. The flag variety $\mathcal{F}_{n}$ has an algebraic and geometric structure as $G / B$, where $B$ is the group of invertible upper triangular matrices, as follows. Given a matrix $g \in G$, we can associate to it the flag $F_{\bullet}$ with $F_{i}$ being the span of the first $i$ columns of $g$. Two matrices $g$ and $g^{\prime}$ represent the same flag if and only if $g^{\prime}=g b$ for some $b \in B$, so complete flags are in one-to-one correspondence with left $B$-cosets of $G$. 
Fix an ordered basis $e_{1}, \ldots, e_{n}$ for $\mathbb{C}^{n}$, and let $E_{\bullet}$ be the flag where $E_{i}$ is the span of the first $i$ basis vectors. Given a permutation $w \in S_{n}$, let the Schubert point $e_{w}$ be the point associated to the $B$-coset of the permutation matrix $w$; its flag $E_{\bullet}^{(w)}$ is the one where $E_{i}^{(w)}=\mathbb{C}\left\{e_{w(1)}, \ldots, e_{w(i)}\right\}$. The rank function $r_{w}$ is defined by

$$
r_{w}(p, q)=\#\{k \leq q \mid w(k) \geq p\} .
$$

The Schubert cell associated to $w$, denoted $X_{w}^{\circ}$, is the subset of $\mathcal{F}_{n}$ corresponding to the set of flags

$$
\left\{F_{\bullet} \mid \underset{F_{q}}{\operatorname{codim}}\left(E_{p} \cap F_{q}\right)=r_{w}(p+1, q) \forall p, q\right\} .
$$

Alternatively, the Schubert cell $X_{w}^{\circ}$ is also the orbit of $e_{w}$ under the left action of the group $B$. The Schubert variety $X_{w}$ is the closure of the Schubert cell $X_{w}^{\circ}$; its points correspond to the flags

$$
\left\{F_{\bullet} \mid \underset{F_{q}}{\operatorname{codim}}\left(E_{p} \cap F_{q}\right) \leq r_{w}(p+1, q) \forall p, q\right\} .
$$

The opposite Schubert cell $\Omega_{w}^{\circ}$ is the subset of $\mathcal{F}_{n}$ corresponding to the set of flags

$$
\left\{F_{\bullet} \mid \operatorname{dim}\left(E_{p}^{\left(w_{0}\right)} \cap F_{q}\right)=r_{w}(p, q) \forall p, q\right\},
$$

or alternatively the orbit of $e_{w}$ under the action of the group $B_{-}$of lower triangular matrices. A lemma of Kazhdan and Lusztig asserts that $e_{v}$ has a neighborhood in $X_{w}$ that is isomorphic to $\mathcal{N}_{v, w} \times \mathbb{C}^{\ell(v)}$, where

$$
\mathcal{N}_{v, w}=\Omega_{v}^{\circ} \cap X_{w}
$$

is known as a Kazhdan-Lusztig variety.

\section{Rothe diagrams of Ici permutations}

Many of the rank conditions in Equation 2.2 are redundant, and Fulton [Ful92] showed that the minimal set of conditions defining any Schubert variety are those from what he called the essential set. The Rothe diagram of $w$ is the set of boxes (which we can think of as being drawn over the permutation matrix)

$$
D(w)=\left\{(p, q) \in \llbracket 1, n \rrbracket \times \llbracket 1, n \rrbracket \mid w(q)<p, w^{-1}(p)>q\right\} .
$$

The diagram can be described visually as follows. For each $q \in \llbracket 1, n \rrbracket$, draw a dot $\bullet$ at $(w(q), q)$. (Coordinates are given in matrix notation.) For each dot draw the "hook" that extends north and east of that dot. The boxes not in any hook are the boxes of the diagram. The essential set $E(w)$ is the set of boxes in $D(w)$ which are northeast corners in some connected component of $D(w)$. To be precise,

$$
E(w)=\{(p, q) \in D(w) \mid(p, q+1) \notin D(w),(p-1, q) \notin D(w)\},
$$

and a matrix $g$ represents a point $g B \in X_{w}$ if and only if the southwest $(n+1-p) \times q$ submatrices of $g$ have rank at most $r_{w}(p, q)$ for all $(p, q) \in E(w)$. Furthermore, $E(w)$ is the minimal subset of $\llbracket 1, n \rrbracket \times \llbracket 1, n \rrbracket$ with this property; no subset of $E(w)$ will correctly define $X_{w}$. (The reader is warned that our conventions for the essential set are different from the original ones of Fulton. [Ful92]) 


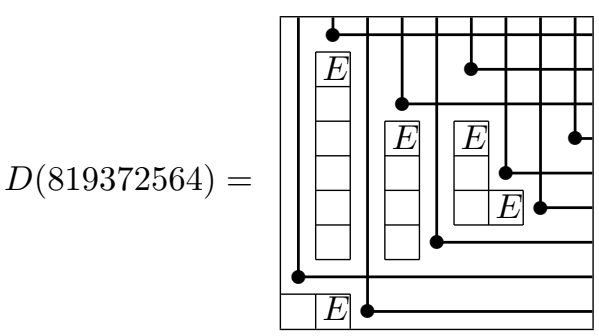

Fig. 1: Diagram and essential set for $w=819372564$.

Example 3.1 Let $w=819372564$. Then the diagram and essential set of $w$ are as in Figure 1 . In particular, $E(w)=\{(2,2),(4,4),(4,6),(6,7),(9,2)\}$.

Let $w \in S_{n}$ be a permutation. We say that $w$ is defined by inclusions if, for each box $(p, q) \in E(w)$, $q-r_{w}(p, q)=\min \{p-1, q\}$. To explain the terminology, note that this condition on the essential set is equivalent to the statement that the intersection conditions defining the Schubert variety are all of the form $E_{p-1} \subset F_{q}$ or $F_{q} \subset E_{p-1}$. Gasharov and Reiner proved the following theorem [GR02, Thm. 4.2].

Theorem 3.2 The following are equivalent:

1. The Schubert variety $X_{w}$ is defined by inclusions.

2. For every box $(p, q) \in E(w)$, either

A: there are no 1's in the permutation matrix $w$ weakly $S W$ of $(p, q)$ (In other words, there is no $k$ such that $k \leq q$ and $w(k) \geq p$.); or

$B$ : there are no 1's in the permutation matrix $w$ strictly $N E$ of $(p, q)$ (In other words, there is no $k$ such that $k>q$ and $w(k)<p$.)

3. The permutation $w$ avoids 4231, 35142, 42513, and 351624 .

We define certain specific technical conditions $\mathrm{W}, \mathrm{X}, \mathrm{Y}$, and $\mathrm{Z}$ regarding the configuration of boxes in the diagram of a permutation $w$. We then say a permutation $w$ is almost defined by inclusions if, for all $(p, q) \in E(w)$, either

- $(p, q)$ satisfies Condition A or Condition B, OR

- $(p, q)$ satisfies both Condition $\mathrm{W}$ or $\mathrm{X}$ and Condition $\mathrm{Y}$ or $\mathrm{Z}$.

Theorem 3.3 If a permutation is not almost defined by inclusions, then it contains one of the patterns 53241, 52341, 52431, 35142, 42513, and 351624 .

Our proof for this theorem follows Gasharov and Reiner's proof for Theorem 3.2 with significant additional complications required in our case.

We also show that by considering the essential set one can canonically associate a permutation defined by inclusions to any permutation almost defined by inclusions. Let $E^{\prime \prime}(w) \subseteq E(w)$ be the subset of the essential set of $w$ consisting of boxes that satisfy neither condition A nor B. 
Theorem 3.4 Let $w$ be a permutation almost defined by inclusions. Then there exists a permutation $v$ such that the essential set $E(v)=E(w) \backslash E^{\prime \prime}(w)$, and the ranks $r_{v}(p, q)=r_{w}(p, q)$ for all $(p, q) \in E(v)$.

These conditions define a unique permutation $v$ which is defined by inclusions. Furthermore, $\ell(v)-$ $\ell(w)$ is the number of boxes in $E^{\prime \prime}(w)$.

\section{Local equations for Ici Schubert varieties}

To show $X_{w}$ is lci whenever $w$ is almost defined by inclusions, we consider explicit equations for $\mathcal{N}_{\text {id, } w}$. Let $S=\mathbb{C}\left[z_{p, q}\right]_{1 \leq q<p \leq n}$. Furthermore, let $M$ be the matrix with 1's on the diagonal, 0's above the diagonal, and $z_{p, q}$ at $(p, q)$.

For any subsets $A$ and $B$ of $\llbracket 1, n \rrbracket$ such that both $A$ and $B$ have the same number of elements, let $d_{A, B}$ denote the minor of $M$ which is the determinant of the square matrix whose rows are the rows of $M$ indexed by elements of $A$ and whose columns are the columns of $M$ indexed by elements of $B$. We will refer to $d_{A, B}$ as a generalized Plücker coordinate.

Given $p, q, r \in \llbracket 1, n \rrbracket$, let $I_{(p, q, r)}^{(v)}$ be the ideal of $S_{v}$ generated by all $d_{A, B}^{(v)}$ where $A \subseteq \llbracket p, n \rrbracket, B \subseteq \llbracket 1, q \rrbracket$, and $\# A=\# B=r+1$; these are all the $r+1$ size minors of the rectangular submatrix consisting of all entries (weakly) SW of $(p, q)$. Given a permutation $w$, let

$$
I_{w}=\sum_{(p, q) \in E(w)} I_{\left(p, q, r_{w}(p, q)\right)} .
$$

The following follows from [WY08, Prop. 3.1], which was first stated in a less concise form in [Ful92].

Proposition 4.1 The Kazhdan-Lusztig variety

$$
\mathcal{N}_{i d, w} \cong \operatorname{Spec} S / I_{w} .
$$

To show that $X_{w}$ is lci whenever $w$ is almost defined by inclusions, we find an explicit generating set for $I_{w}$ with $\left(\begin{array}{l}n \\ 2\end{array}\right)-\ell(w)$ polynomials (rather than the significantly larger number of polynomials a priori required by 4.1 .

First suppose $w$ is defined by inclusions. Let $k$ be the number of boxes in $E(w)$. Fix a total ordering of the essential set $E(w)$ in which smaller rank boxes come before larger rank boxes, and label the boxes of the essential set $\left(p_{1}, q_{1}\right), \ldots,\left(p_{k}, q_{k}\right)$ according to this ordering. Let $r_{m}=r_{w}\left(p_{m}, q_{m}\right)$ for each $m \in \llbracket 1, k \rrbracket$. By our ordering $r_{i} \leq r_{j}$ if $i<j$. Also let $R_{m} \subseteq D(w)$ be the subset of the diagram consisting of all boxes which are SW of $\left(p_{m}, q_{m}\right)$ but not SW of $p_{m^{\prime}}, q_{m^{\prime}}$ for any $m^{\prime}<m$. Each region $R_{m}$ turns out to be a rectangle consisting of boxes in the same connected component of the diagram.

For each box $(x, y) \in D(w)$, we define a polynomial $f_{(x, y)}$ in $S$ (which will be a generalized Plücker coordinate) as follows. If $r_{w}(x, y)=0$, then let

$$
A(x, y)=\{x\} \text { and } B(x, y)=\{y\} .
$$

Otherwise, the box $(x, y)$ is in some rectangle $R_{m}$. Let $A(x, y)=\llbracket p_{m}, p_{m}+r_{m}-1 \rrbracket \cup\left\{x+r_{m}\right\}$, and let $B(x, y)=\left\{y-r_{m}\right\} \cup \llbracket q_{m}-r_{m}+1, q_{m} \rrbracket$. Now let

$$
f_{(x, y)}=d_{A(x, y), B(x, y)} \text { and } J_{w}=\left\langle f_{(x, y)}\right\rangle_{(x, y) \in D(w)} .
$$

We show the following, noting that $\operatorname{codim} X_{w}=\# D(w)$. 
Theorem 4.2 Suppose $w$ is defined by inclusions. Then the ideals $I_{w}$ and $J_{w}$ are equal. Hence $I_{w}$ defines a local complete intersection.

Now we consider the general case where $w$ is almost defined by inclusions. Given $(p, q) \in E^{\prime \prime}(w)$, let $A^{\prime}(p, q)=\llbracket p, p+r_{w}(p, q) \rrbracket$ and $B^{\prime}(p, q)=\llbracket q-r_{w}(p, q), q \rrbracket$. Define

$$
f_{(p, q)}=d_{A^{\prime}(p, q), B^{\prime}(p, q)}
$$

We show the following.

Theorem 4.3 Let $w$ be almost defined by inclusions, $v$ the defined by inclusions permutation associated to $w$ by Theorem 3.4 and let $(p, q) \in E^{\prime \prime}(w)$. Then

$$
I_{w}=I_{v}+\left\langle f_{(p, q)}\right\rangle_{(p, q) \in E^{\prime \prime}(w)} .
$$

Theorems 4.2 and 4.3 are proven by explicit manipulation of determinants.

Combining the statements of this section and the previous shows the following.

Theorem 4.4 Suppose $w$ avoids 52431, 52341, 53241, 35142, 42513, and 351624. Then $X_{w}$ is a local complete intersection.

\section{Mesh patterns and non-Ici Schubert varieties}

To show the converse to Theorem 4.4 we need some additional generalizations of pattern avoidance.

Interval patterns were introduced by Yong and the second author in [WY08] and, we now recall their definition. First recall that the Bruhat order on the symmetric group is the reflexive transitive closure of the partial order defined by declaring $u$ to be less than or equal to $v$ if $v=u s_{i j}$ and $\ell(v)>\ell(u)$. Here $s_{i j}$ is the transposition that switches the (not necessarily adjacent) positions $i$ and $j$, and $\ell(v)$ is the number of inversions in the permutation $v$, or equivalently, the length of any reduced expression for $v$ as a product of simple reflections $s_{i(i+1)}$, called the Coxeter length of $v$. We use the symbol " $\leqslant$ " to denote the Bruhat order. Now, if $[u, v]$ and $[x, w]$ are intervals in the Bruhat orders on $S_{m}$ and $S_{n}$ respectively, we say that $[u, v]$ (interval) pattern embeds in $[x, w]$ if there is a common embedding consisting of indices $i_{1}<\cdots<i_{m}$ of $u$ in $x$ and $v$ in $w$, such that the entries of $x$ and $w$ outside of these indices agree, and additionally, the intervals $[u, v]$ and $[x, w]$ are isomorphic posets. Since, given $u, v, w$, and the indices of the embedding, the permutation $x$ is automatically determined, we can omit $x$ in the notation. Hence we will abuse terminology to say that $[u, v]$ embeds in $w$ or that $w$ avoids $[u, v]$ as appropriate.

The motivation for these patterns is that they govern any "reasonable" local property, as shown by Yong and the second author [WY08, Thm 2.6]. In particular, if the Kazhdan-Lusztig variety $\mathcal{N}_{u, v}$ is not lci and $[u, v]$ embeds in a permutation $w$, then $X_{w}$ is not lci. We identify two infinite families and eleven isolated intervals $[u, v]$ such that the Kazhdan-Lusztig variety $\mathcal{N}_{u, v}$ is not lci. We show that, if $w$ contains one of the six given patterns, then $w$ will interval contain either one of the eleven intervals or an interval from one of the two infinite families.

The two infinite families and eleven isolated intervals are as follows:

Family A consists of intervals of the form $[(a+1) a \cdots 1(a+b+2) \cdots(a+2),(a+b+2)(a+$ 1) $a \cdots 2(a+b+1) \cdots(a+2) 1]$, where $a, b>0$ and $a>1$ or $b>1$. We list the first few members of the family in Figure 2. 


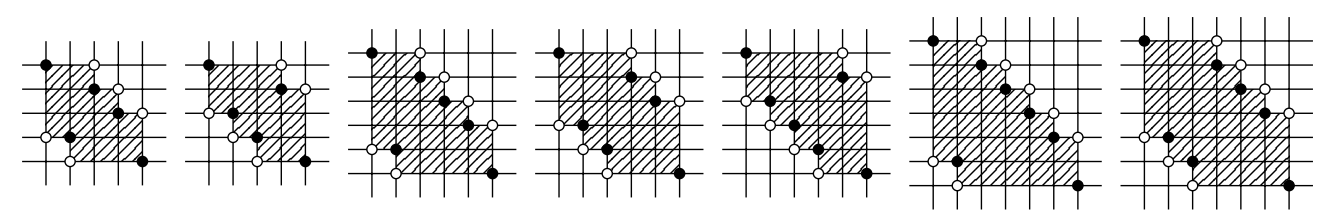

Fig. 2: The first few members of the family $A$.

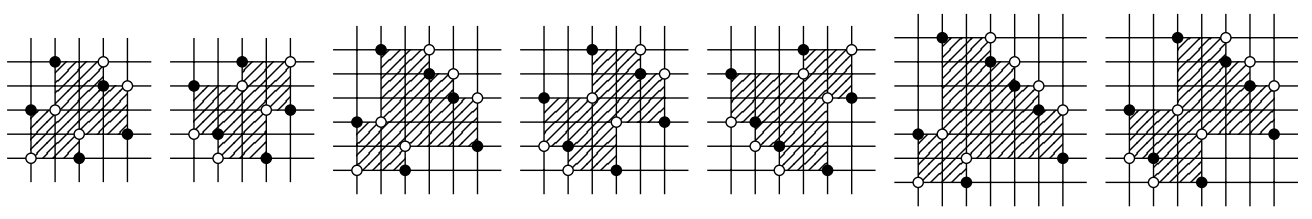

Fig. 3: The first few members of the family $B$.

Family B consists of intervals of the form $[(a+1) \cdots 1(a+3)(a+2)(a+b+4) \cdots(a+4),(a+$ $3)(a+1) \cdots 2(a+b+4) 1(a+b+3) \cdots(a+4)(a+2)]$, where $a, b \geq 0$ and $a+b \geq 1$. We list the first few members of the family in Figure 3

We list the exceptional intervals in Figure 4.

The varieties $\mathcal{N}_{u, v}$ for the infinite families $\mathrm{A}$ and $\mathrm{B}$ define portions of the singular locus (as shown independently by Billey-Warrington [BW03], Cortez [Cor03], Kassel-Lascoux-Reutenauer [KLR03], and Manivel [Man01b]), and their equations were determined independently by Cortez [Cor03] and Manivel [Man01a]. For the eleven isolated intervals, minimal defining equations for $\mathcal{N}_{u, v}$ can be calculated explicitly by hand, showing they are not lci.

Hence to prove the converse to Theorem 4.4, it suffices to show the following.

Theorem 5.1 If the permutation $w$ contains one of the patterns 53241, 52341, 52431, 35142, 42513, and 351624, then $w$ contains an interval from Family A, an interval from Family B, or one of the eleven intervals in Figure 4.

Interval patterns are difficult to work with directly, so we translate them using [Ú11, Lemma 22] into mesh patterns, as implied by the figures above. A mesh pattern is a pair $(v, R)$ where $v$ is a permutation (classical pattern) from $S_{m}$ and $R$ is a subset of the square $\llbracket 0, m \rrbracket \times \llbracket 0, m \rrbracket$. An embedding of $(v, R)$ in a permutation $w$ is first of all an embedding of $v$ in $w$ in the usual sense, meaning indices $i_{1}<\cdots<i_{m}$ such that the relative order of $w\left(i_{1}\right), \ldots, w\left(i_{m}\right)$ is given by $v$. Equivalently, we have order-preserving bijections $\alpha, \beta: \llbracket 1, m \rrbracket \rightarrow \llbracket 1, n \rrbracket$ such that

$$
\{(\alpha(i), \beta(j)) \mid(i, j) \in G(v)\} \subseteq G(w),
$$

where for any permutation $u, G(u)$ is defined to be the graph

$$
G(u)=\{(i, u(i)): i \in \llbracket 1, n \rrbracket\}
$$



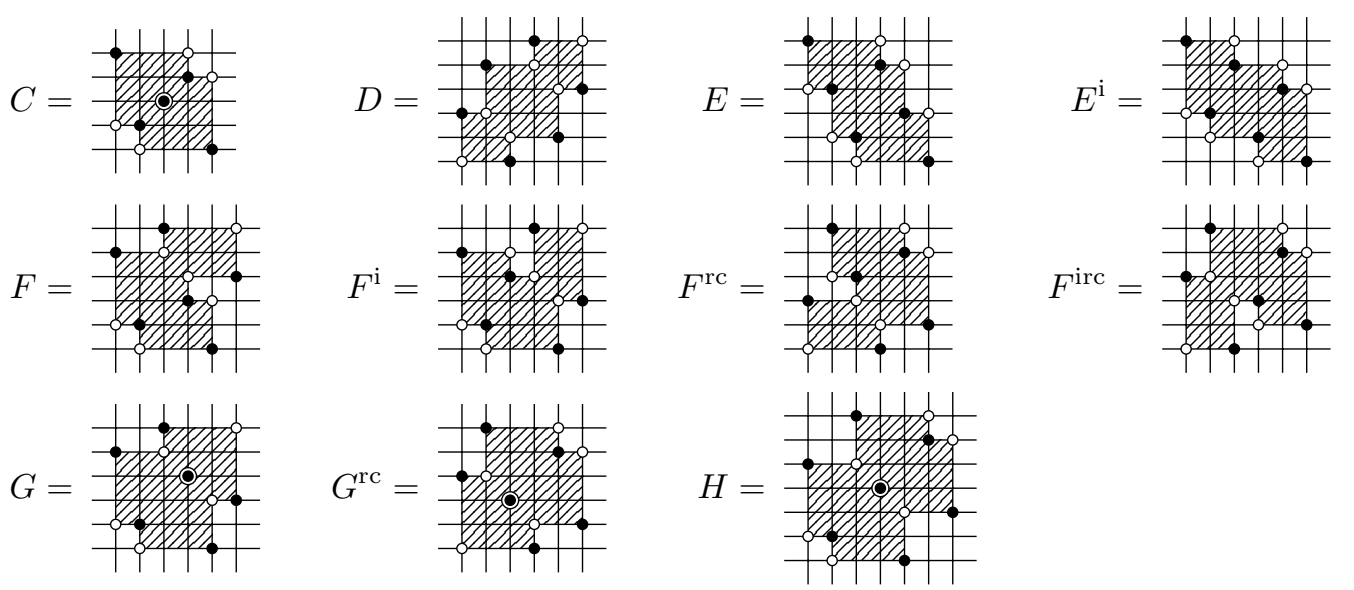

Fig. 4: The exceptional intervals.

of $u$. In addition, to be an embedding of $(v, R)$, we further require the following:

$$
\text { If }(i, j) \in R \text { then } R_{i j} \cap G(u)=\emptyset .
$$

Here $R_{i j}$ is defined as the rectangle $\llbracket \alpha(i)+1, \alpha(i+1)-1 \rrbracket \times \llbracket \beta(j)+1, \beta(j+1)-1 \rrbracket$, where, as a convention, we set $\alpha(0)=0=\beta(0)$ and $\alpha(m+1)=n+1=\beta(m+1)$.

To further simplify the proof, we also use the notion of marked mesh pattern, originally given by the first author [Ú11, Subsec. 4.1]. Marked mesh patterns extend the definition of mesh patterns by allowing another kind of designated regions where a certain number of elements is required to be present. We only review their definition via an example:

Example 5.2 To show that the marked mesh pattern occurs in the permutation 526413, we first need to find an occurrence of the underlying classical pattern 132. There are three such occurrences, as shown below.
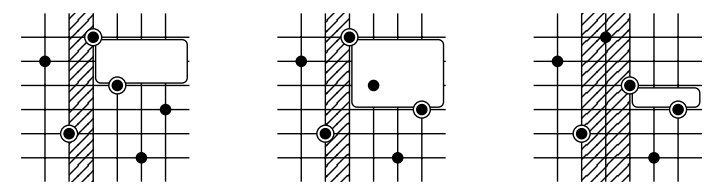

However, only the middle occurrence of 132 is an occurrence of the marked mesh pattern since it is the only occurrence having at least one dot in the box marked with "1" in the pattern, as well as having no dots in the shaded vertical strip.

\section{Singularity implications from patterns}

Various properties of Schubert varieties have been characterized by patterns. Figure 5 shows how these various properties imply each other. These implications are known on general geometric principles, but 

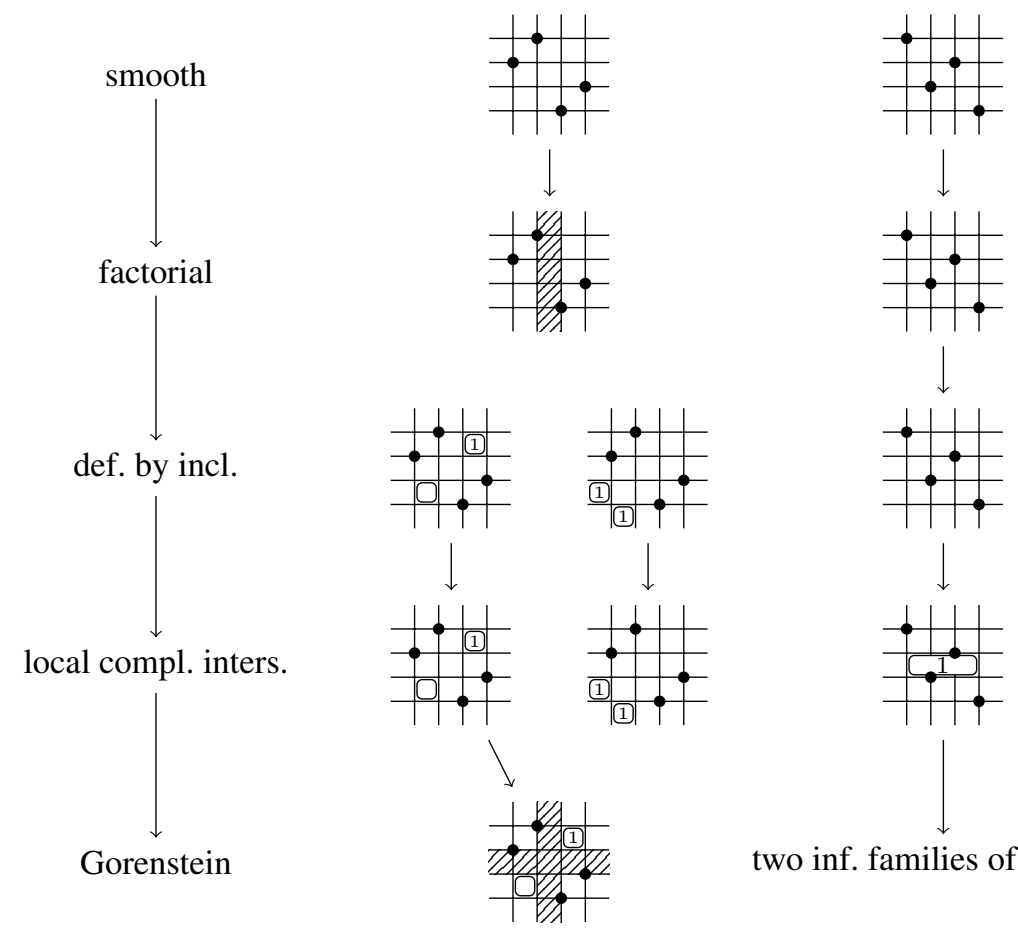

two inf. families of patterns

Fig. 5: Properties of Schubert varieties described with pattern avoidance. An arrow between two patterns means that avoidance of the first pattern implies avoidance of the second.

they can also be proven combinatorially. Notice that this diagram shows that the two classical patterns 3421 and 4231 characterizing smooth varieties each lead to distinct groups of patterns for less restrictive criteria.

\section{Kostant polynomials at the identity}

From our explicit equations for the lci Schubert varieties in a neighborhood of the identity, we obtain the following formulas for particular specializations of the double Schubert polynomials of Lascoux and Schützenberger [LS82a, LS82b], as these represent local cohomology classes at the identity.

Corollary 7.1 Suppose $X_{v}$ is defined by inclusions. Then

$$
\mathfrak{S}_{w_{0} v}\left(t_{1}, \ldots, t_{n} ; t_{n}, \ldots, t_{1}\right)=\prod_{(x, y) \in D(v)}\left(t_{y-r_{v}(x, y)}-t_{x+r_{v}(x, y)}\right) .
$$

Corollary 7.2 Suppose $X_{w}$ is lci, and let $v$ be the permutation defined by inclusions associated to $w$ by 
Theorem 3.4 Then

$$
\mathfrak{S}_{w_{0} w}\left(t_{1}, \ldots, t_{n} ; t_{n}, \ldots, t_{1}\right)=\mathfrak{S}_{w_{0} v}\left(t_{1}, \ldots, t_{n} ; t_{n}, \ldots, t_{1}\right) \prod_{(p, q) \in E^{\prime \prime}(w)}\left(\sum_{i=0}^{r_{w}(p, q)} t_{q-i}-t_{p+i}\right) .
$$

For $j$ and $i$ with $1 \leq j<i \leq n$, let $s_{j i} \in S_{n}$ be the transposition switching $j$ and $i$. For the case where $X_{v}$ is smooth, the following is a theorem of Kumar [Kum96], restated in our language. (The equivalence of our statement with the original is unfortunately folklore; parts of the connection can be found in [BL00, Gol01].)

Theorem 7.3 The Schubert variety $X_{v}$ is smooth if and only if

$$
\mathfrak{S}_{w_{0} v}\left(t_{1}, \ldots, t_{n} ; t_{n}, \ldots, t_{1}\right)=\prod_{(i, j): s_{j i} \leq v}\left(t_{j}-t_{i}\right)
$$

Similar statements hold in all three cases for analogous specializations of double Grothendieck polynomials.

Comparing Theorem 7.3 and Corollary 7.1 tells us (because $\mathbb{Q}\left[t_{1}, \ldots, t_{n}\right]$ is a unique factorization domain) that, in the case where $X_{v}$ is smooth, the map

$$
\phi: D(v) \rightarrow\left\{(i, j) \mid s_{j i} \not \leq v\right\} \quad(x, y) \mapsto\left(x+r_{v}(x, y), y-r_{v}(x, y)\right)
$$

is a bijection. Indeed, $D(v)$ always has $\left(\begin{array}{c}n \\ 2\end{array}\right)-\ell(v)$ elements, and a theorem of Carrell [Car94] states that $\left\{(i, j) \mid s_{j i} \not \leq v\right\}$ has $\left(\begin{array}{c}n \\ 2\end{array}\right)-\ell(v)$ elements whenever $X_{v}$ is smooth. We believe a purely combinatorial proof can be given that $\phi$ is a bijection whenever $v$ avoids 4231 and 3412 .

In addition, the map $\phi$ can be defined for any $v$ if we allow the codomain to include all transpositions (or equivalently all positive roots). We conjecture the image of $\phi$ always contains $\left\{(i, j) \mid s_{j i} \not \leq v\right\}$ and equals this set precisely when $v$ is defined by inclusions.

These considerations may help in finding a connection between the results of Gasharov and Reiner and those of Hultman, Linusson, Shareshian, and Sjöstrand.

\section{Questions}

We conclude with a list of questions for future research. The first two are purely combinatorial problems.

Question 8.1 Enumerate the permutations $w \in S_{n}$ for which $X_{w}$ is lci. An ideal answer would provide an explicit generating function.

For the smooth case, the analogous question was answered in unpublished work of Haiman [Hai92]. (A proof of this formula appears in [BMB07].) Bousquet-Mélou and Butler [BMB07] gave a generating function for the number of factorial Schubert varieties. On the other hand, the analogous question for Schubert varieties defined by inclusions and for Gorenstein Schubert varieties are still open.

We expect that a generating function for the Schubert varieties defined by inclusions could possibly be obtained by an argument similar to the one for smooth Schubert varieties. Answering the following more specific combinatorial question may help in deriving the generating function enumerating lci Schubert varieties from a (currently unknown) generating function for Schubert varieties defined by inclusions. 
Question 8.2 Determine if the converse to Theorem 3.4 is true. More precisely, suppose $w$ is a permutation with essential set $E(w)$, and suppose $E^{\prime \prime}(w) \subset E(w)$ is the set of essential set boxes that are not defined by inclusions. If $E(w) \backslash E^{\prime \prime}(w)$ is the essential set for some permutation $v$ (necessarily defined by inclusions) such that $r_{v}(p, q)=r_{w}(p, q)$ for all $(p, q) \in E(w) \backslash E^{\prime \prime}(w)$ and $\ell(v)-\ell(w)=\# E^{\prime \prime}(w)$,

then is $w$ necessarily almost defined by inclusions (or equivalently lci)?

Another question is the following.

Question 8.3 Determine if $X_{w}$ being lci depends solely on the Bruhat graph of $w$. If so, find reasonable properties of the Bruhat graph that characterize when $X_{w}$ is lci.

Note that it is a theorem of Carrell [Car94] that $X_{w}$ is smooth (for simply-laced types) if and only if the Bruhat graph is regular.

\section{References}

[BL00] Sara Billey and V. Lakshmibai. Singular loci of Schubert varieties, volume 182 of Progress in Mathematics. Birkhäuser Boston Inc., Boston, MA, 2000.

[BMB07] Mireille Bousquet-Mélou and Steve Butler. Forest-like permutations. Ann. Comb., 11(34):335-354, 2007.

[BW03] Sara C. Billey and Gregory S. Warrington. Maximal singular loci of Schubert varieties in SL $(n) /$ B. Trans. Amer. Math. Soc., 355(10):3915-3945 (electronic), 2003.

[Car94] James B. Carrell. The Bruhat graph of a Coxeter group, a conjecture of Deodhar, and rational smoothness of Schubert varieties. In Algebraic groups and their generalizations: classical methods (University Park, PA, 1991), volume 56 of Proc. Sympos. Pure Math., pages 53-61. Amer. Math. Soc., Providence, RI, 1994.

[Cor03] Aurélie Cortez. Singularités génériques et quasi-résolutions des variétés de Schubert pour le groupe linéaire. Adv. Math., 178(2):396-445, 2003.

[Ful92] William Fulton. Flags, Schubert polynomials, degeneracy loci, and determinantal formulas. Duke Math. J., 65(3):381-420, 1992.

[Gol01] R. F. Goldin. The cohomology ring of weight varieties and polygon spaces. Adv. Math., 160(2):175-204, 2001.

[GR02] V. Gasharov and V. Reiner. Cohomology of smooth Schubert varieties in partial flag manifolds. J. London Math. Soc. (2), 66(3):550-562, 2002.

[Hai92] Mark Haiman. Smooth schubert varieties. preprint, 1992.

[HLSS09] Axel Hultman, Svante Linusson, John Shareshian, and Jonas Sjöstrand. From Bruhat intervals to intersection lattices and a conjecture of Postnikov. J. Combin. Theory Ser. A, 116(3):564$580,2009$.

[KLR03] Christian Kassel, Alain Lascoux, and Christophe Reutenauer. The singular locus of a Schubert variety. J. Algebra, 269(1):74-108, 2003. 
[Kum96] Shrawan Kumar. The nil Hecke ring and singularity of Schubert varieties. Invent. Math., 123(3):471-506, 1996.

[LS82a] Alain Lascoux and Marcel-Paul Schützenberger. Polynômes de Schubert. C. R. Acad. Sci. Paris Sér. I Math., 294(13):447-450, 1982.

[LS82b] Alain Lascoux and Marcel-Paul Schützenberger. Structure de Hopf de l'anneau de cohomologie et de l'anneau de Grothendieck d'une variété de drapeaux. C. R. Acad. Sci. Paris Sér. I Math., 295(11):629-633, 1982.

[LS90] V. Lakshmibai and B. Sandhya. Criterion for smoothness of Schubert varieties in $\mathrm{Sl}(n) / B$. Proc. Indian Acad. Sci. Math. Sci., 100(1):45-52, 1990.

[Man01a] L. Manivel. Generic singularities of Schubert varieties. arXiv:math/0105239v1 [math.AG], 2001.

[Man01b] L. Manivel. Le lieu singulier des variétés de Schubert. Internat. Math. Res. Notices, (16):849$871,2001$.

[Ú11] Henning Úlfarsson. A unification of permutation patterns related to Schubert varieties. Pure Math. Appl. to appear, 2011.

[UW11] Henning Úlfarsson and Alexander Woo. Which Schubert varieties are local complete intersections? Preprint. arXiv:1111.6146v1 [math.AG], 2011.

[WY06] Alexander Woo and Alexander Yong. When is a Schubert variety Gorenstein? Adv. Math., 207(1):205-220, 2006.

[WY08] Alexander Woo and Alexander Yong. Governing singularities of Schubert varieties. J. Algebra, 320(2):495-520, 2008. 